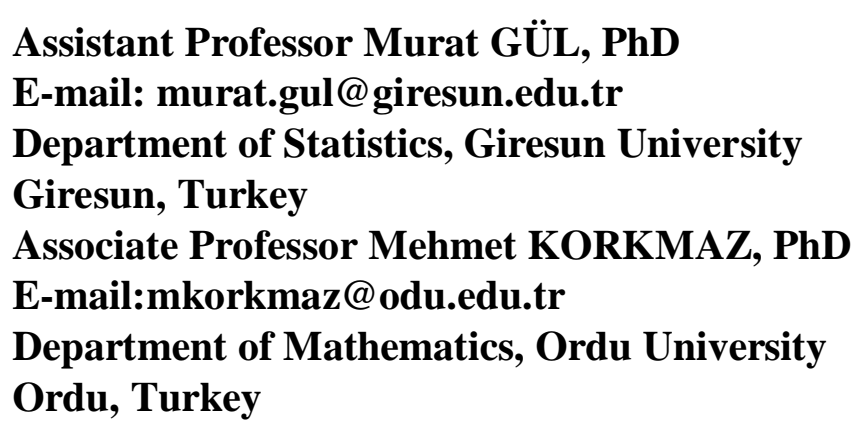

\title{
THE EFFECT OF ASYMMETRIC AND SYMMETRIC DEPENDENCE IN SOME GROWTH MODELS USING COPULAS
}

\begin{abstract}
Growth models are included in non-linear regression models. On the other hand, dependence is one of the most important issues of statistics. Many models have been applied to data and variables which have dependent and correlated. However, the effect of dependence on growth models has not been revealed in researches. Therefore, the aim of this study is to demonstrate the effect of dependence on growth models. Dependence structure can be in the form of asymmetric and symmetric dependence. From this point on, the effect of asymmetric and symmetrical dependence on growth models is shown. For this, a simulation study is considered. In the simulation study, correlated variables are produced by means of Copula functions. The dependence on the variables generated by Copulas is constructed as weak, medium, strong. These levels (weak,medium,strong) of dependence that are constructed in variables have affected the $R$-squared $\left(R^{2}\right)$ and parameters of growth models. According to the results of simulation study and growth models, in particular, the Von Bertalanffy model has performed better, when the asymmetric dependence is 0.8 (strongdependence). The Brody model comes into prominence in symmetric dependence 0.8 (strong-dependence), while the Logistic model comes into prominence in symmetric dependence 0.2 (weak-dependence). This study will give researchers remarkable information about which model to apply when working with strong, medium, weak correlated data
\end{abstract}

Keywords: Copula, Growth Models, Correlated Data, AsymmetricSymmetric Dependence, Non-Linear Regression.

JEL Classification: C02, C15, C63 


\section{Introduction}

Correlated data have been treated using different models because it has different data structures such as multivariate observations, clustered data, repeated measurements, longitudinal data, spatial correlated data, etc (Tsuyuguchi et al,2019). Due to this structure of the data, many models have been developed to work with correlated data and variables. Correlated data and variables are included in the model, the parameters of the model have been estimated, the model's explanatory power is looked at, and the model is tried to find out if it is meaningful. As with other models, correlated data and variable structure are also available in growth models. (English et al,2005; Gwaze et al,2002;Zwaigenbaum et al,2014).In this context, growth models may have much or little correlation or dependence between the independent and dependent variable.

There is a wide range of models in the literature that work by taking into account the effect of correlated data and variables. The main models of these are the following; Ridge regression is a robust method that can be modeled highly correlated data. (Chiang et al,2018). Generalized linear mixed models have been broadly applied to the study of correlated data in plenty of research fields. The linear mixed model with normal errors has been a well-known model for the examination of repeated measures and longitudinal data (Kang,2020). The popularity and flexibility of these models are due to their modeling of withinsubject correlation that appears in repeated measured data.

Generalized additive partial linear models (GAPLM) for analyzing correlated data are especially used in statistical methods (Manghi et al,2019). Generalized additive partial linear models are an important approach for modeling correlation in clustered, repeated measurement and longitudinal data. These models have parametric and nonparametric components that offer correlation structure and marginal distributions together. Multilevel modeling is a statistical approach that is often used to analyze correlated data, such as data that is correlated within individuals or groups, variables (Xanthakis et al,2013).

Correlation variables are encountered in many studies. Therefore, it is important to generate correlated variables. The generation of correlated variables is carried out by copula models, which are a new phenomenon. Now, practitioners and researchers are able to generate variables with correlations at the rate they want, thanks to copula models.The importance of this approach is modeling and capturing dependencies among random variables, regardless of the form of the marginal distribution. The main advantage of the application of the Copula approach is that it can be modeled flexibly without knowing the distribution function. Correlation measures reflect the degree of dependence but not the structure and shape of dependence. Copula models allow for multivariate distributions based on marginal distributions that have not the same distribution family furthermore, marginal distributions are heterogeneous. Thus, the researcher gets rid of taking into account only the present multivariate distributions. Copulas 
The Effect of Asymmetric and Symmetric Dependence in some Growth Models Using Copulas

happened to be extremely convenient devices for examining the dependence construction between random variables, such as symmetric and asymmetric dependence and tail dependence. Copulas will be invariant under nonlinear on the rise transformations of random variables.

There are a few measures of dependence, such as Kendall's tau $(\tau)$ and Spearman's rho with this property they offer a suitable option to linear correlation. These dependence measures are called rank correlation or correspondence measures and they grab nonlinear dependence between random variables. Correlation measures reflect the degree of dependence but do not reflect the structure and the shape of the dependence. In this sense, the copulas reveal the degree of dependence between two or more random variables and the structure of the dependence. When we look at the fields of statistics and data sciences where the copulas are used ,the dependence structure of multivariate distributions, obtaining and demonstrating new multivariate distributions, developing dependence measures between new variables and simulation studies requiring a dependence between variables are frequently used in the generation of correlated data.

Although correlated data and variables are used in many models, there is no study showing the effect of correlated variables using copulas in growth models. In this respect, it is thought that this article will fill this gap.

Because regression modeling is performed to reveal the relationship between the dependent and the independent variable, there may be a dependence or correlation between the dependent and the independent variable. The aim of this study is to generate the dependence between variables by using copulas; when there is a correlation between the dependent and the independent variable, that is, when there is dependence; to find R-squared $\left(R^{2}\right)$ by estimating the parameters of growth models that fit the data; to understand which model performs better in the strong, weak, medium correlation between the dependent variable and independent variable. Then to discuss the effect of asymmetric and symmetrical dependence on growth models based on the dependence between variables.

The results of our study will give an idea of which model to apply when working with strong, medium, weak correlated data from every discipline to practitioners and researchers when they want to use growth models. It will also constitute a new perspective on the fact that before constructing the growth model, they should be more careful when selecting variables, thinking about the effect of dependence.

The sections of the study are structured as follows. Materials and Methods in the second section, Application and Results in the third section, Conclusion in the fourth section are given.

DOI: $10.24818 / 18423264 / 55.1 .21 .15$ 


\section{Materials and Methods}

\subsection{Some Growth Models}

In this study, the Logistic, Gompertz, Monomolecular (Brody) and von Bertalanffy models widely used are introduced shortly as a mathematical formulation

\subsubsection{Logistic Model}

The origin of the logistic growth model is described by the following equation (1):

$\frac{\partial \mathrm{y}}{\partial \mathrm{x}}=C y\left(1-\frac{y}{A}\right)$

where $\mathrm{y}$ is the value of growth, $\mathrm{x}$ is time, $\mathrm{C}$ is the proportion about growth, $\mathrm{A}$ is maximum sustainable growth. This differential equation produces the model called the logistic model in Equation 2:

$\mathrm{y}=\frac{A}{1+B e^{-C x}}$

where B is a constant. The logistic model in Equation 2 is found in the following operations:

Separating the variables in Equation 1 we get: $\frac{\text { Ady }}{\mathrm{y}(\mathrm{A}-\mathrm{y})}=C d x$ Integrating both sides we get:

$\int \frac{\mathrm{Ady}}{\mathrm{y}(\mathrm{A}-\mathrm{y})}=\int C d x \quad ; \int\left(\frac{1}{y}+\frac{1}{A-y}\right) d y=\int C d x \quad ; \ln (y)-\ln (A-y)=C x+C_{1}$

$\ln ((A-y) / y)=-C x-C_{1} \quad$,where $C_{1}$ is constant. Taking the exponential of both sides we get: $(A-y) / y=B e^{-C x} ; \frac{A}{y}-1=B e^{-C x}$, where $B=e^{-C_{1}}$, After this we get: $\frac{A}{y}=B e^{-C x}+1$ and then get the logistic model as

$$
y=\frac{A}{1+B e^{-C x}}
$$

\subsubsection{Gompertz Model}

The origin of the Gompertz growth model is described by the following equation (3):

$\frac{\partial \mathrm{y}}{\partial \mathrm{x}}=\operatorname{Cy} \ln \left(\frac{A}{y}\right)$

where $y$ is the value of growth, $\mathrm{x}$ is time, $\mathrm{C}$ is the proportion about growth, $\mathrm{A}$ is maximum sustainable growth

This differential equation produces the model called the Gompertz model in equation 4: 
The Effect of Asymmetric and Symmetric Dependence in some Growth Models Using Copulas

$\mathrm{y}=A e^{-B e^{-C x}}$

where B is a constant. The Gompertz model in Equation 4 is found in the following operations:

Separating the variables in Equation 3 we get: $\frac{\mathrm{dy}}{\operatorname{yln}\left(\frac{A}{y}\right)}=C d x$

Integrating both sides we get:

$\int \frac{\mathrm{dy}}{\mathrm{y} \ln \left(\frac{A}{y}\right)}=\int C d x \quad ;$ If we say $\mathrm{u}=\ln \left(\frac{A}{y}\right) \quad$ and $d u=\frac{-A / y^{2}}{A / y} d y=\frac{-d y}{y}$, then we can get $-\int \frac{\mathrm{du}}{\mathrm{u}}=\int C d x$ and $-\ln u=C x+C_{2}$

Taking the exponential of both sides we get; $u=B e^{-C x} ; \ln \left(\frac{A}{y}\right)=B e^{-C x}$, where $B=e^{-C_{2}}$ Again taking the exponential of both sides we get: $\frac{A}{y}=e^{B e^{-C x}}$ and then get the Gompertz model as

$$
\mathrm{y}=A e^{-B e^{-C x}}
$$

\subsubsection{Monomolecular (Brody) Model}

Since the origin of the monomolecular growth model suggested that the growth is directly proportional to the difference of the maximum sustainable constant A and the growth, this growth model starts the following differential equation:

$\frac{\partial \mathrm{y}}{\partial \mathrm{x}}=C(A-y)$

where $\mathrm{y}$ is the value of growth, $\mathrm{x}$ is time, $\mathrm{C}$ is the proportion about growth, $\mathrm{A}$ is maximum sustainable growth

This differential equation produces a model of the following form:

$\mathrm{y}=A\left(1-B e^{-C x}\right)$

where B is a constant. The monomolecular model in Equation 6 is found the following operations: Separating the variables in Equation 5 we get:

$\frac{\mathrm{dy}}{\mathrm{A}-\mathrm{y}}=C d x$ Integrating both sides we get: $-\ln (A-y)=C x+C_{1}, \ln (A-y)^{-1}=$ $C x+C_{1}$ where $C_{1}$ is constant. Taking the exponential of both sides we get:

DOI: 10.24818/18423264/55.1.21.15 
Murat Gül, Mehmet Korkmaz

$\overline{(A-y)^{-1}}=P e^{C x} ; \frac{1}{A-y}=P e^{C x} ;$ where $P=e^{C_{1}}$, Multiply both sides by A-y we get:

$1=A P e^{C x}-y P e^{C x} ; y P e^{C x}=A P e^{C x}-1$, Divide by $\mathrm{M} e^{C x}$ we get a monomolecular model as

$y=A\left(1-B e^{-C x}\right)$, where $B=1 / A P$

\subsubsection{Von Bertalanffy Model}

Von Bertalanffy model is formed by using a special case of the Bernoulli differential equation, namely:

$\frac{\mathrm{dy}}{\mathrm{dx}}=v y^{2 / 3}-y K^{1 / 3}$

where $\mathrm{y}$ is the value of growth, $\mathrm{x}$ is time, $\mathrm{v}$ is the proportion about growth, $\mathrm{K}$ is the value about maximum sustainable growth

This differential equation produces a model of the following form:

$\mathrm{y}=A\left(1-B e^{-C x}\right)^{3}$

where $\mathrm{B}$ is a constant, $\mathrm{A}$ is maximum sustainable growth, $\mathrm{C}$ is the proportion about growth. The von Bertalanffy model in Equation 8 is found in the following operations:

Separating the variables in Equation 7 we get: $\frac{d y}{y^{2 / 3}\left(v-y^{1 / 3} K^{1 / 3}\right)}=d x$

Integrating both sides we get:

$\int \frac{d y}{y^{2 / 3}\left(v-y^{1 / 3} K^{1 / 3}\right)}=\int d x ; \quad$ if we say $\mathrm{u}=\left(v-y^{\frac{1}{3}} K^{\frac{1}{3}}\right)$ and $d \mathrm{u}=$ $-\frac{1}{3} y^{-\frac{2}{3}} K^{\frac{1}{3}} d y$ then we can get $\int \frac{-3 K^{-1 / 3} d u}{u}=x+c$ and then $-3 K^{-\frac{1}{3}} \ln |u|=x+$ $c, \ln |u|=\frac{-x}{3 K^{-1 / 3}}+c$, Taking the exponential of both sides we get:

$u=e^{\frac{-x}{3 K^{-1 / 3}+c}} ; v-y^{\frac{1}{3}} K^{\frac{1}{3}}=e^{\frac{-x}{3 K^{-1 / 3}+c}} ; v-M e^{\frac{-K^{1 / 3} x}{3}}=y^{1 / 3} K^{1 / 3} ;$ where $M=e^{c}$ and then we get:

$\left(v-M e^{\frac{-K^{\frac{1}{3}} x}{3}}\right)^{3}=y K ; \frac{1}{K}\left(v\left(1-\frac{M}{v} e^{\frac{-K^{\frac{1}{3}} x}{3}}\right)^{3}=y ; y=\frac{v^{3}}{K}\left(1-\frac{M}{v} e^{\frac{-K^{\frac{1}{3}} x}{3}}\right)^{3}\right.$

and then we get the von Bertalanffy model as 
The Effect of Asymmetric and Symmetric Dependence in some Growth Models Using Copulas

$\bar{y}=A\left(1-B e^{-C x}\right)^{3}$

where $A=\frac{V^{3}}{K}, B=\frac{M}{v}$ and $C=\frac{K^{1 / 3}}{3}$

\subsection{Copulas}

\subsubsection{Definition and Properties of Copula}

$C:[0,1]^{2} \rightarrow[0,1]$, function is called the copula when it provides the following properties.

1. $C(0, v)=C(u, 0)=0 ; u, v \in[0,1]$

2. $C(1, v)=C(u, 1)=u ; u, v \in[0,1]$

3. $a_{1} \leq a_{2} ; b_{1} \leq b_{2} ; a_{1}, a_{2}, b_{1}, b_{2} \in[0,1]$,

$C\left(a_{1}, b_{1}\right)+C\left(a_{2}, b_{2}\right)-C\left(a_{1}, b_{2}\right)-C\left(a_{2}, b_{1}\right) \geq 0$

Theorem 2.1. Sklar's Theorem: this theorem is the building block of the copula theory. This theorem explains what copula does in the relationship between multidimensional distribution functions and univariate marginalities (Nelsen,2006). There is only one $\mathrm{C}$ copula for a n-dimensional distribution function $F$ given by continuous marginal distribution functions $F_{1}, F_{2}, \ldots F_{n}$ and $x_{i} \in R, i=1,2, \ldots, \mathrm{n}$. $F\left(x_{1}, x_{2}, \ldots, x_{n}\right)=C\left(F_{1}\left(x_{1}\right), F_{2}\left(x_{2}\right), \ldots F_{n}\left(x_{n}\right)\right)$

is expressed as in (9). If $F_{1}, F_{2}, \ldots F_{n}$ is not continuous, $\mathrm{C}$ is identified on $\operatorname{RanF}_{1} x \operatorname{RanF}_{2} x \ldots x \operatorname{RanF}_{n}$ in one form. Conversely, if $\mathrm{C}$ is a copula and $F_{1}, F_{2}, \ldots F_{n}$ are distribution functions, then the function $\mathrm{F}$ defined in Equation 9 is a joint distribution function with marginal $F_{1}, F_{2}, \ldots F_{n}$ (Nelsen,2006).

This theorem provides both a structured plan and a parameterization of multivariate distributions for copula. Indeed, for a multivariate $F$ function given with $F_{1}, F_{2}, \ldots F_{n}$ marginals, $u_{i} \in[0,1], i=1,2, \ldots, \mathrm{n}$.

$C\left(u_{1}, u_{2}, \ldots, u_{n}\right)=F\left(F_{1}^{-1}\left(u_{1}\right), F_{2}^{-1}\left(u_{2}\right), \ldots F_{n}^{-1}\left(u_{n}\right)\right)$

is given. Here the left side of equation 10 is a copula automatically (Nelsen,2006; Malevergne \& Sornette, 2003).

Since copula functions indicate the dependence structure between random variables, dependence measures can be expressed with the help of the copula. Therefore, it can be considered that the copula shows the dependence structure of

DOI: 10.24818/18423264/55.1.21.15 
the data and a multivariate distribution if appropriate, that is, if it is correctly selected.

\subsubsection{Concepts of Dependence}

Kendall's tau $(\tau)$ and Spearman's rho $(\rho)$ can be estimated over the copula families. This can be carried out by the following equations.

$\tau\left(X_{1}, X_{2}\right)=4 \iint C(u, v) d C(u, v)-1$

Spearman's $\rho$ can be calculated by the following equation

$\rho_{X, Y}=12 \iint C(u, v) d u d v-3$

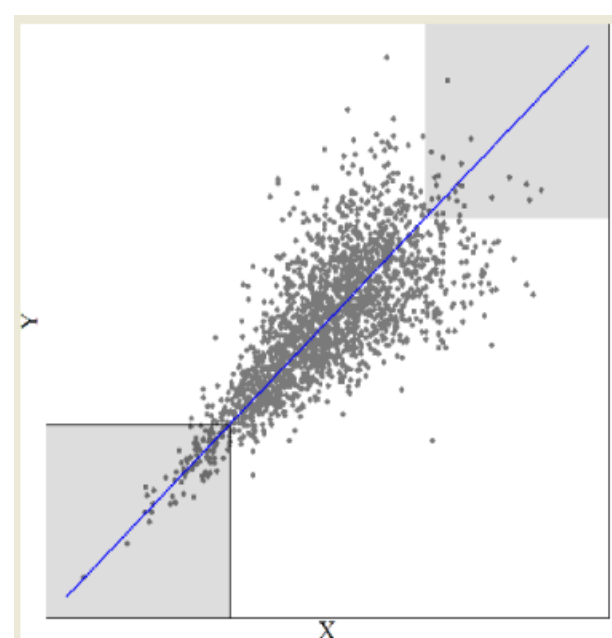

(a) Asymmetric Dependence

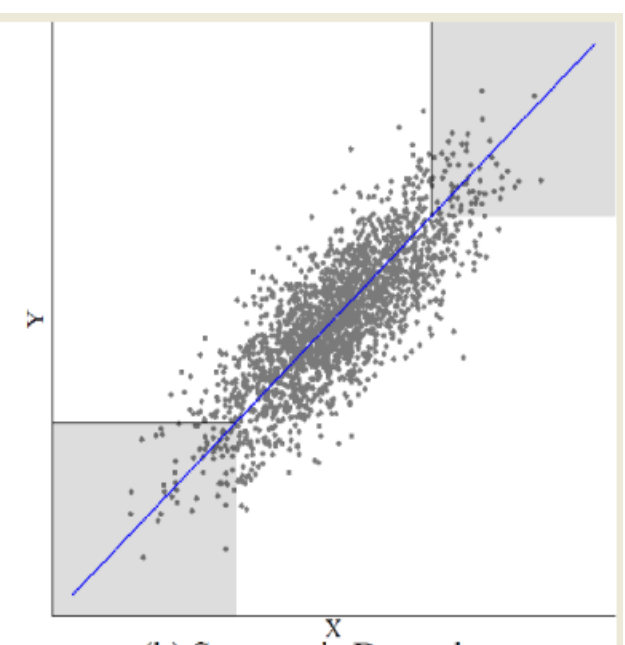

(b) Symmetric Dependence

Figure-1. Scatter Plot for Asymmetric Dependence and Symmetric Dependence (Jiang et al,2014).

In asymmetric dependence, the points $\mathrm{X}$ and $\mathrm{Y}$ are scattered in the right tail, while in the left tail, the points $\mathrm{X}$ and $\mathrm{Y}$ are tighter, which means they are dense. Therefore, the dependence on the left tail is stronger than the right tail dependence. Clayton Copula can be used to achieve asymmetric dependence. In symmetric dependence, the $\mathrm{X}$ and $\mathrm{Y}$ points in the right and left tail are of the same density in both tails, because they are in a symmetric structure. Therefore, the left and right tail dependence are equal. Gaussian (Normal) Copula can be used to obtain symmetric dependence.

\subsubsection{Clayton Copula}


The Effect of Asymmetric and Symmetric Dependence in some Growth Models Using Copulas

Clayton Copula is an asymmetric copula that is used to model asymmetric dependence. The left tail shows greater dependence than the right tail. The Clayton Capula is defined as follows.

$C_{\theta}(u, v)=\max \left(\left[u^{-\theta}+v^{-\theta}-1\right]^{-1 / \theta}, 0\right)$

This copula relies upon a single parameter $\theta$ with support in $[0, \infty)$.

The dependence between the observations increases as the value of $\theta$ increases, with $\theta \rightarrow 0$ implying, $\lim _{n \rightarrow \infty} C_{\theta}(u, v)=u v=\Pi(u, v)$ independence copula and $\theta \rightarrow \infty$ implying perfect dependence (Arnold, 2006).

\subsubsection{Gaussian (Normal) Copula}

The Gaussian Copula is derived from the multivariate Gaussian distribution. $\Phi_{\Sigma}^{n}$ denotes the joint distribution function of the $\mathrm{n}$-variate standard normal distribution function with linear correlation matrix $\Sigma$, and $\Phi^{-1}$ denotes the inverse of the distribution function of the univariate standard normal distribution. In this case, the n-variate Gaussian Copula $C_{\Sigma}^{\Phi}$ is defined as follows.

$$
C_{\Sigma}^{\Phi}\left(u_{1}, \ldots . ., u_{n}\right)=\Phi_{\Sigma}^{n}\left(\Phi^{-1}\left(u_{1}\right), \ldots . ., \Phi^{-1}\left(u_{n}\right)\right), \quad \forall\left(u_{1}, \ldots, u_{n}\right) \in[0,1]^{n}
$$

In the bivariate case, the copula expression can be written as

$$
C_{\rho_{12}}^{\Phi}(u, v)=\int_{-\infty}^{\Phi^{-1}(u)} \int_{-\infty}^{\Phi^{-1}(v)} \frac{1}{2 \pi\left(1-\rho_{12}^{2}\right)^{1 / 2}} \exp \left(-\frac{s^{2}-2 \rho_{12} \cdot s \cdot t+t^{2}}{2\left(1-\rho_{12}^{2}\right)}\right) d s d t, \quad(\mathrm{u}, \mathrm{v}) \in[0,1]^{2}
$$

Note that $\rho_{12}$ is simply the usual linear correlation coefficient of the corresponding bivariate normal distribution. Since Gaussian Copulas are radially symmetric copula that is used to model symmetric dependence, the coefficients of right and left tail dependence are equal. (Embrechts et al,2003).

\section{Application and Results}

The Clayton Copula is used to represent asymmetric dependence, while the Gaussian Copula is used to represent symmetric dependence. We compare the performance of four growth models (Logistic, Gompertz, Brody, Von Bertalanffy) for simulated data which have dependence weak, medium, strong (correlated) using

DOI: 10.24818/18423264/55.1.21.15 
copulas. Simulation results obtained for $N=15,25,50,100,150,300$ and $R^{2}$, parameters $(\mathrm{A}, \mathrm{B}, \mathrm{C})$ of growth models averaged over 50 simulations.

\subsection{Simulation Study}

A simulation is planned for this part of the study. According to this simulation study

Step-1:X,Y pairs are produced by means of the copula, according to a certain dependence (weak, medium, strong ) and sample size.

Step-2: The obtained pairs are put into Logistic, Gompertz, Brody, Von Bertalanffy models.

Step-3: The mean values of the $\mathrm{R}^{2}$ and parameters attained from these models are reported according to the sample size.

A simulation study is conducted to investigate the performance of growth models for

sample sizes and dependence. We consider two cases for dependence. In one case, $X$ and $Y$ are asymmetric dependence variables, and in the other, $\mathrm{X}$ and $\mathrm{Y}$ are symmetric dependence variables and the $\mathrm{R}^{2}$ and parameters of growth models obtained from the simulation study are shown in Table 1, Table 2, Table 3.

Table 1. For Growth Models, Simulation Results when dependence is 0.5

\begin{tabular}{lllll}
\hline & Logistic & Gompertz & Brody & Von Bertalanfy \\
\hline Asymmetric & $R^{2}=0.62368$ & $R^{2}=0.62038$ & $R^{2}=0.59685$ & $R^{2}=0.62190$ \\
Dependence & $\mathbf{A}=0.87614$ & $\mathbf{A}=1.42010$ & $\mathbf{A}=1.32856$ & $\mathbf{A}=1.29567$ \\
Kendall Tau=0.5 & $\mathbf{B}=3.13991$ & $\mathbf{B}=1.21207$ & $\mathbf{B}=1.09254$ & $\mathbf{B}=0.90248$ \\
Clayton Capula & $\mathbf{C}=11.77614$ & $\mathbf{C}=5.24586$ & $\mathbf{C}=2.75305$ & $\mathbf{C}=4.88395$ \\
N=15 & & & & \\
\hline Symmetric & $R^{2}=0.58557$ & $R^{2}=0.58560$ & $R^{2}=0.56262$ & $R^{2}=0.59294$ \\
Dependence & $\mathbf{A}=1.09631$ & $\mathbf{A}=1.42568$ & $\mathbf{A}=1.66724$ & $\mathbf{A}=1.54426$ \\
Kendall Tau=0.5 & $\mathbf{B}=3.43636$ & $\mathbf{B}=2.26582$ & $\mathbf{B}=4.76500$ & $\mathbf{B}=3.34600$ \\
Gaussian & $\mathbf{C}=20.99780$ & $\mathbf{C}=18.03560$ & $\mathbf{C}=11.27890$ & $\mathbf{C}=9.29254$ \\
N=15 & & & & \\
\hline Asymmetric & $R^{2}=0.52891$ & $R^{2}=0.54555$ & $R^{2}=0.53320$ & $R^{2}=0.547302$ \\
Dependence & $\mathbf{A}=0.92139$ & $\mathbf{A}=0.89095$ & $\mathbf{A}=1.28143$ & $\mathbf{A}=1.15330$ \\
Kendall Tau=0.5 & $\mathbf{B}=2.60874$ & $\mathbf{B}=1.28982$ & $\mathbf{B}=1.16705$ & $\mathbf{B}=0.76179$ \\
Clayton Copula & $\mathbf{C}=9.46448$ & $\mathbf{C}=5.55045$ & $\mathbf{C}=2.45991$ & $\mathbf{C}=4.11577$ \\
N=25 & & & & \\
\hline Symmetric & $R^{2}=0.57203$ & $R^{2}=0.57076$ & $R^{2}=0.55974$ & $R^{2}=0.56417$ \\
Dependence & $\mathbf{A}=1.06513$ & $\mathbf{A}=1.54550$ & $\mathbf{A}=1.90585$ & $\mathbf{A}=1.56706$ \\
Kendall Tau=0.5 & $\mathbf{B}=2.26448$ & $\mathbf{B}=1.04115$ & $\mathbf{B}=1.06741$ & $\mathbf{B}=0.33217$ \\
Gaussian & $\mathbf{C}=7.00000$ & $\mathbf{C}=4.11906$ & $\mathbf{C}=2.10837$ & $\mathbf{C}=3.25554$ \\
N=25 & & & & \\
\hline
\end{tabular}


The Effect of Asymmetric and Symmetric Dependence in some Growth Models Using Copulas

\begin{tabular}{lllll}
\hline Asymmetric & $R^{2}=0.52406$ & $R^{2}=0.52318$ & $R^{2}=0.52022$ & $R^{2}=0.53153$ \\
Dependence & $\mathbf{A}=0.74604$ & $\mathbf{A}=0.80770$ & $\mathbf{A}=0.96694$ & $\mathbf{A}=0.78647$ \\
Kendall Tau=0.5 & $\mathbf{B}=2.09761$ & $\mathbf{B}=1.0073$ & $\mathbf{B}=1.02290$ & $\mathbf{B}=0.68193$ \\
Clayton Copula & $\mathbf{C}=8.07267$ & $\mathbf{C}=5.35308$ & $\mathbf{C}=2.56249$ & $\mathbf{C}=4.52522$ \\
N=50 & & & & \\
\hline Symmetric & $R^{2}=0.49131$ & $R^{2}=0.49628$ & $R^{2}=0.47896$ & $R^{2}=0.49021$ \\
Dependence & $\mathbf{A}=1.49162$ & $\mathbf{A}=1.45922$ & $\mathbf{A}=1.66428$ & $\mathbf{A}=1.44600$ \\
Kendall Tau=0.5 & $\mathbf{B}=1.76577$ & $\mathbf{B}=0.75594$ & $\mathbf{B}=0.92764$ & $\mathbf{B}=0.09394$ \\
Gaussian & $\mathbf{C}=3.56900$ & $\mathbf{C}=2.16722$ & $\mathbf{C}=0.97007$ & $\mathbf{C}=1.60406$ \\
N=50 & & & & \\
\hline Asymmetric & $R^{2}=0.50544$ & $R^{2}=0.50868$ & $R^{2}=0.50990$ & $R^{2}=0.51020$ \\
Dependence & $\mathbf{A}=0.72390$ & $\mathbf{A}=0.75604$ & $\mathbf{A}=0.87246$ & $\mathbf{A}=0.90122$ \\
Kendall Tau=0.5 & $\mathbf{B}=1.86024$ & $\mathbf{B}=0.89388$ & $\mathbf{B}=0.99808$ & $\mathbf{B}=0.60559$ \\
Clayton Copula & $\mathbf{C}=6.92664$ & $\mathbf{C}=4.67666$ & $\mathbf{C}=2.29308$ & $\mathbf{C}=3.84994$ \\
N=100 & & & & \\
\hline Symmetric & $R^{2}=0.48021$ & $R^{2}=0.48361$ & $R^{2}=0.50063$ & $R^{2}=0.48425$ \\
Dependence & $\mathbf{A}=1.43449$ & $\mathbf{A}=1.49244$ & $\mathbf{A}=2.07189$ & $\mathbf{A}=1.74895$ \\
Kendall Tau=0.5 & $\mathbf{B}=1.68323$ & $\mathbf{B}=0.74134$ & $\mathbf{B}=0.93744$ & $\mathbf{B}=0.53488$ \\
Gaussian & $\mathbf{C}=3.17660$ & $\mathbf{C}=1.86112$ & $\mathbf{C}=0.75770$ & $\mathbf{C}=1.42070$ \\
N=100 & & & &
\end{tabular}

From Table 1,for asymmetric dependence, $\mathrm{R}^{2}$ values are high for all used models in asymmetric dependence 0.5 (medium-dependence) and sample size $\mathrm{N}=15$. While the sample size is increasing, $\mathrm{R}^{2}$ values decrease gradually for all models used. $\mathrm{R}^{2}$ is highest in the Logistic model in asymmetric dependence 0.5(mediumdependence) and sample size $\mathrm{N}=15 . \mathrm{R}^{2}$ is highest in the Von Bertalanffy model in sample size $\mathrm{N}=25,50,100$ in asymmetric dependence 0.5 (medium-dependence), while the highest $R^{2}$ is seen in Brody model in sample size $N=150,300$ in asymmetric dependence 0.5 (medium-dependence).

Table 1 (Continued). For Growth Models, Simulation Results when dependence is 0.5

\begin{tabular}{lllll}
\hline & Logistic & Gompertz & Brody & Von Bertalanffy \\
\hline Asymmetric & $R^{2}=0.49140$ & $R^{2}=0.49560$ & $R^{2}=0.49812$ & $R^{2}=0.49731$ \\
Dependence & $\mathbf{A}=0.73140$ & $\mathbf{A}=0.76588$ & $\mathbf{A}=0.89268$ & $\mathbf{A}=0.79204$ \\
Kendall Tau=0.5 & $\mathbf{B}=1.81180$ & $\mathbf{B}=0.86076$ & $\mathbf{B}=1.00154$ & $\mathbf{B}=0.59120$ \\
Clayton Copula & $\mathbf{C}=6.55728$ & $\mathbf{C}=4.37110$ & $\mathbf{C}=2.19616$ & $\mathbf{C}=3.64663$ \\
N=150 & & & & \\
\hline Symmetric & $R^{2}=0.47960$ & $R^{2}=0.48372$ & $R^{2}=0.48783$ & $R^{2}=0.48443$ \\
Dependence & $\mathbf{A}=1.40802$ & $\mathbf{A}=1.80831$ & $\mathbf{A}=2.38204$ & $\mathbf{A}=2.36864$ \\
Kendall Tau=0.5 & $\mathbf{B}=1.71845$ & $\mathbf{B}=0.79517$ & $\mathbf{B}=0.93438$ & $\mathbf{B}=0.55777$ \\
Gaussian & $\mathbf{C}=2.95574$ & $\mathbf{C}=1.61435$ & $\mathbf{C}=0.68967$ & $\mathbf{C}=1.19611$ \\
N=150 & & & & \\
\hline
\end{tabular}

DOI: 10.24818/18423264/55.1.21.15 
Murat Gül, Mehmet Korkmaz

\begin{tabular}{lllll}
\hline Asymmetric & $R^{2}=0.48146$ & $R^{2}=0.48572$ & $R^{2}=0.48910$ & $R^{2}=0.48712$ \\
Dependence & $\mathbf{A}=0.73162$ & $\mathbf{A}=0.76368$ & $\mathbf{A}=0.86842$ & $\mathbf{A}=0.78352$ \\
Kendall Tau=0.5 & $\mathbf{B}=1.67278$ & $\mathbf{B}=0.78814$ & $\mathbf{B}=0.96782$ & $\mathbf{B}=0.55686$ \\
Clayton Copula & $\mathbf{C}=6.00496$ & $\mathbf{C}=4.09722$ & $\mathbf{C}=2.12272$ & $\mathbf{C}=3.45876$ \\
N=300 & & & & \\
\hline Symmetric & $R^{2}=0.47412$ & $R^{2}=0.47692$ & $R^{2}=0.48067$ & $R^{2}=0.47573$ \\
Dependence & $\mathbf{A}=1.44486$ & $\mathbf{A}=2.17990$ & $\mathbf{A}=6.47267$ & $\mathbf{A}=3.43900$ \\
Kendall Tau=0.5 & $\mathbf{B}=1.76506$ & $\mathbf{B}=0.84931$ & $\mathbf{B}=0.95500$ & $\mathbf{B}=0.58533$ \\
Gaussian & $\mathbf{C}=2.47231$ & $\mathbf{C}=1.19088$ & $\mathbf{C}=0.28333$ & $\mathbf{C}=0.79176$ \\
N=300 & & & &
\end{tabular}

From Table 1, for symmetric dependence, $\mathrm{R}^{2}$ values are high for all used models in symmetric dependence 0.5 (medium-dependence) and sample size $\mathrm{N}=15$. While the sample size is increasing, $\mathrm{R}^{2}$ values tend to decrease gradually for all models used with a few exceptions.

It is the Von Bertalanffy model whose $\mathrm{R}^{2}$ is highest in symmetric dependence 0.5 (medium-dependence) and sample size $\mathrm{N}=15$. Logistic is the model that its $\mathrm{R}^{2}$ is highest in symmetric dependence 0.5 (medium-dependence) and sample size $\mathrm{N}=25 . \mathrm{R}^{2}$ is highest in the Gompertz model in sample size $\mathrm{N}=50$ in symmetric dependence 0.5(medium-dependence), while the highest $\mathrm{R}^{2}$ is seen in the Brody model in sample size $\mathrm{N}=100,150,300$ in symmetric dependence 0.5 (medium-dependence).

Table 2. For Growth Models, Simulation Results when dependence is 0.8

\begin{tabular}{lllll}
\hline & Logistic & Gompertz & Brody & Von Bertalanffy \\
\hline Asymmetric & $R^{2}=0.90042$ & $R^{2}=0.90406$ & $R^{2}=0.89510$ & $R^{2}=0.903756$ \\
Dependence & $\mathbf{A}=0.96526$ & $\mathbf{A}=1.09400$ & $\mathbf{A}=2.42529$ & $\mathbf{A}=1.24790$ \\
Kendall Tau=0.8 & $\mathbf{B}=2.50640$ & $\mathbf{B}=1.19018$ & $\mathbf{B}=1.03736$ & $\mathbf{B}=0.74084$ \\
Clayton Copula & $\mathbf{C}=5.66654$ & $\mathbf{C}=3.30063$ & $\mathbf{C}=0.90731$ & $\mathbf{C}=2.49704$ \\
N=15 & & & & \\
\hline Symmetric & $R^{2}=0.90376$ & $R^{2}=0.91016$ & $R^{2}=0.91730$ & $R^{2}=0.91085$ \\
Dependence & $\mathbf{A}=1.17340$ & $\mathbf{A}=1.48320$ & $\mathbf{A}=2.92244$ & $\mathbf{A}=1.94490$ \\
Kendall Tau $=0.8$ & $\mathbf{B}=2.57074$ & $\mathbf{B}=1.22265$ & $\mathbf{B}=1.00637$ & $\mathbf{B}=0.71081$ \\
Gaussian & $\mathbf{C}=5.07044$ & $\mathbf{C}=2.77459$ & $\mathbf{C}=0.61400$ & $\mathbf{C}=1.82273$ \\
N=15 & & & & \\
\hline Asymmetric & $R^{2}=0.88292$ & $R^{2}=0.88524$ & $R^{2}=0.87914$ & $R^{2}=0.88550$ \\
Dependence & $\mathbf{A}=0.94240$ & $\mathbf{A}=1.09688$ & $\mathbf{A}=2.68955$ & $\mathbf{A}=1.24856$ \\
Kendall Tau $=0.8$ & $\mathbf{B}=2.45492$ & $\mathbf{B}=1.16534$ & $\mathbf{B}=1.01993$ & $\mathbf{B}=0.72286$ \\
Clayton Copula & $\mathbf{C}=5.48844$ & $\mathbf{C}=3.11654$ & $\mathbf{C}=0.69700$ & $\mathbf{C}=2.31108$ \\
N=25 & & & & \\
\hline & & & & \\
\hline
\end{tabular}


The Effect of Asymmetric and Symmetric Dependence in some Growth Models Using Copulas

\begin{tabular}{|c|c|c|c|c|}
\hline Symmetric & $R^{2}=0.90158$ & $R^{2}=0.90540$ & $R^{2}=0.91128$ & $R^{2}=0.90679$ \\
\hline Dependence & $\mathbf{A}=1.08618$ & $\mathbf{A}=1.46776$ & $\mathbf{A}=3.04272$ & $\mathbf{A}=1.738938$ \\
\hline Kendall Tau $=0.8$ & $\mathbf{B}=2.36556$ & $\mathbf{B}=1.10906$ & $\mathbf{B}=1.00104$ & $B=0.68377$ \\
\hline $\begin{array}{l}\text { Gaussian Copula } \\
\mathrm{N}=25\end{array}$ & $C=4.52666$ & $\mathbf{C}=2.34358$ & $\mathrm{C}=0.48672$ & $\mathrm{C}=1.657042$ \\
\hline Asymmetric & $R^{2}=0.89266$ & $R^{2}=0.89580$ & $R^{2}=0.89050$ & $R^{2}=0.89634$ \\
\hline Dependence & $\mathbf{A}=1.13750$ & $A=1.09602$ & $\mathbf{A}=2.50945$ & $\mathbf{A}=1.23806$ \\
\hline Kendall Tau=0.8 & $\mathbf{B}=2.40006$ & $\mathbf{B}=1.29280$ & $\mathbf{B}=1.01323$ & $\mathbf{B}=0.69680$ \\
\hline $\begin{array}{l}\text { Clayton Copula } \\
\mathrm{N}=50\end{array}$ & $\mathbf{C}=5.23978$ & $\mathbf{C}=2.94378$ & $\mathbf{C}=0.60389$ & $\mathbf{C}=2.16484$ \\
\hline Symmetric & $R^{2}=0.88388$ & $R^{2}=0.88780$ & $R^{2}=0.89326$ & $R^{2}=0.88904$ \\
\hline Dependence & $\mathbf{A}=1.09534$ & $\mathbf{A}=1.42396$ & $\mathbf{A}=4.53673$ & $\mathbf{A}=1.86506$ \\
\hline Kendall Tau $=0.8$ & $\mathbf{B}=2.26288$ & $\mathbf{B}=1.06116$ & $\mathbf{B}=0.99789$ & $B=0.66540$ \\
\hline $\begin{array}{l}\text { Gaussian Copula } \\
\mathrm{N}=50\end{array}$ & $\mathbf{C}=4.20700$ & $C=2.16108$ & $\mathbf{C}=0.36479$ & $\mathbf{C}=1.46718$ \\
\hline Asymmetric & $R^{2}=0.89484$ & $R^{2}=0.89828$ & $R^{2}=0.89825$ & $R^{2}=0.89898$ \\
\hline Dependence & $\mathbf{A}=0.94446$ & $\mathbf{A}=1.07942$ & $\mathbf{A}=2.71927$ & $\mathbf{A}=1.19542$ \\
\hline Kendall Tau $=0.8$ & $\mathbf{B}=2.35194$ & $\mathbf{B}=1.10798$ & $\mathbf{B}=1.01058$ & $\mathbf{B}=0.68726$ \\
\hline $\begin{array}{l}\text { Clayton Copula } \\
\mathrm{N}=100\end{array}$ & $\mathbf{C}=5.13066$ & $\mathbf{C}=2.90972$ & $\mathbf{C}=0.57083$ & $\mathbf{C}=2.15442$ \\
\hline
\end{tabular}

From Table 2, for asymmetric dependence, $\mathrm{R}^{2}$ is high for all used models in asymmetric dependence 0.8 (strong-dependence) and sample size $\mathrm{N}=15$, while the sample size is increasing, the $\mathrm{R}^{2}$ value exhibits a fluctuating case for all models used.

The highest $\mathrm{R}^{2}$ is seen in the Von Bertalanffy model in asymmetric dependence 0.8 (strong-dependence) and sample size $\mathrm{N}=25, \mathrm{~N}=50, \mathrm{~N}=100, \mathrm{~N}=150$ and $\mathrm{N}=300$, while only in the sample size $\mathrm{N}=15$, the highest $\mathrm{R}^{2}$ is seen in the Gompertz model with very little difference.

Table 2 (Continued). For Growth Models, Simulation Results when dependence is 0.8

\begin{tabular}{lllll}
\hline & Logistic & Gompertz & Brody & Von Bertalanffy \\
\hline Asymmetric & $R^{2}=0.89086$ & $R^{2}=0.89504$ & $R^{2}=0.89533$ & $R^{2}=0.89612$ \\
Dependence & $\mathbf{A}=0.94126$ & $\mathbf{A}=1.07324$ & $\mathbf{A}=2.62047$ & $\mathbf{A}=1.18584$ \\
Kendall Tau=0.8 & $\mathbf{B}=2.36272$ & $\mathbf{B}=1.11078$ & $\mathbf{B}=1.00769$ & $\mathbf{B}=0.68504$ \\
Clayton Copula & $\mathbf{C}=5.16504$ & $\mathbf{C}=2.91406$ & $\mathbf{C}=0.52527$ & $\mathbf{C}=2.14624$ \\
N=150 & & & & \\
\hline Symmetric & $R^{2}=0.88770$ & $R^{2}=0.89242$ & $R^{2}=0.89689$ & $R^{2}=0.89382$ \\
Dependence & $\mathbf{A}=1.08976$ & $\mathbf{A}=1.36724$ & $\mathbf{A}=6.03695$ & $\mathbf{A}=1.65156$ \\
Kendall Tau=0.8 & $\mathbf{B}=2.22514$ & $\mathbf{B}=1.04066$ & $\mathbf{B}=0.99668$ & $\mathbf{B}=0.65460$ \\
Gaussian Copula & $\mathbf{C}=4.07934$ & $\mathbf{C}=2.09810$ & $\mathbf{C}=0.19716$ & $\mathbf{C}=1.42242$ \\
N=150 & & & & \\
\hline
\end{tabular}

DOI: 10.24818/18423264/55.1.21.15 
Murat Gül, Mehmet Korkmaz

\begin{tabular}{lllll}
\hline Asymmetric & $R^{2}=0.88834$ & $R^{2}=0.89232$ & $R^{2}=0.89252$ & $R^{2}=0.89308$ \\
Dependence & $\mathbf{A}=0.93102$ & $\mathbf{A}=1.05422$ & $\mathrm{~A}=2.31780$ & $\mathrm{~A}=1.15732$ \\
Kendall Tau=0.8 & $\mathbf{B}=2.35130$ & $\mathbf{B}=1.10888$ & $\mathrm{~B}=1.00896$ & $\mathrm{~B}=0.68688$ \\
Clayton Copula & $\mathbf{C}=5.20674$ & $\mathbf{C}=2.96482$ & $\mathrm{C}=0.56420$ & $\mathrm{C}=2.19808$ \\
$\mathrm{~N}=300$ & & & & \\
\hline Symmetric & $R^{2}=0.88890$ & $R^{2}=0.89372$ & $R^{2}=0.89366$ & $R^{2}=0.89526$ \\
Dependence & $\mathbf{A}=1.09298$ & $\mathbf{A}=1.36678$ & $\mathbf{A}=6.31175$ & $\mathbf{A}=1.64226$ \\
Kendall Tau=0.8 & $\mathbf{B}=2.24218$ & $\mathbf{B}=1.04760$ & $\mathbf{B}=0.99717$ & $\mathbf{B}=0.65722$ \\
Gaussian Copula & $\mathbf{C}=4.07078$ & $\mathbf{C}=2.08638$ & $\mathbf{C}=0.18542$ & $\mathbf{C}=1.40968$ \\
N=300 & & & & \\
\hline
\end{tabular}

From Table 2, for symmetric dependence, $\mathrm{R}^{2}$ is high for all used models in symmetric dependence 0.8 (strong-dependence) and the sample size $\mathrm{N}=15$, while $\mathrm{R}^{2}$ is decreased for all models until sample size $N=50$. The $R^{2}$ valueexhibits a fluctuating case for all models used while the sample size is increasing after $\mathrm{N}=50$. The highest $\mathrm{R}^{2}$ is seen in the Brody model in symmetric dependence 0.8(strongdependence) and sample size $\mathrm{N}=15, \mathrm{~N}=25, \mathrm{~N}=50, \mathrm{~N}=100$ and $\mathrm{N}=150$, while only in the sample size $\mathrm{N}=300$, the highest $\mathrm{R}^{2}$ is seen in the Von Bertalanffy model with very little difference.

Table 3. For Growth Models, Simulation Results when dependence is 0.2

\begin{tabular}{lllll}
\hline & Logistic & Gompertz & Brody & Von Bertalanffy \\
\hline Asymmetric & $R^{2}=0.19082$ & $R^{2}=0.18750$ & $R^{2}=0.17142$ & $R^{2}=0.17480$ \\
Dependence & & & & \\
Kendall Tau=0.2 & & & & \\
Clayton Capula & & & & \\
N=15 & & & & \\
\hline Symmetric & $R^{2}=0.19863$ & $R^{2}=0.18972$ & $R^{2}=0.15447$ & $R^{2}=0.16834$ \\
Dependence & & & & \\
Kendall Tau=0.2 & & & & \\
Gaussian Copula & & & & \\
N=15 & & $R^{2}=0.14952$ & $R^{2}=0.13910$ & $R^{2}=0.13864$ \\
\hline Asymmetric & $R^{2}=0.15306$ & & & \\
Dependence & & & & \\
Kendall Tau=0.2 & & & & \\
Clayton Copula & & & & \\
N=25 & & & & \\
\hline $\begin{array}{l}\text { Symmetric } \\
\text { Dependence } \\
\text { Kendall Tau=0.2 }\end{array}$ & & & & \\
Gaussian Copula & & & & \\
N=25 & & & & \\
\hline
\end{tabular}


The Effect of Asymmetric and Symmetric Dependence in some Growth Models Using Copulas

\begin{tabular}{lllll}
\hline Asymmetric & $R^{2}=0.15140$ & $R^{2}=0.15044$ & $R^{2}=0.14628$ & $R^{2}=0.13806$ \\
Dependence & & & & \\
Kendall Tau=0.2 & & & & \\
Clayton Copula & & & & \\
N=50 & & & & \\
\hline Symmetric & $R^{2}=0.14053$ & $R^{2}=0.14003$ & $R^{2}=0.13316$ & $R^{2}=0.12726$ \\
Dependence & & & & \\
Kendall Tau=0.2 & & & & \\
Gaussian Copula & & & & \\
N=50 & & & & \\
\hline Asymmetric & $R^{2}=0.12506$ & $R^{2}=0.12574$ & $R^{2}=0.12492$ & $R^{2}=0.11712$ \\
Dependence & & & & \\
Kendall Tau=0.2 & & & & \\
Clayton Copula & & & & \\
N=100 & & & & \\
\hline Symmetric & $R^{2}=0.11207$ & $R^{2}=0.10824$ & $R^{2}=0.10386$ & $R^{2}=0.11182$ \\
Dependence & & & & \\
Kendall Tau=0.2 & & & & \\
Gaussian Copula & & & & \\
N=100 & & & & \\
\hline From Table 3, for asymmetric & & & \\
\end{tabular}

From Table 3, for asymmetric dependence, $\mathrm{R}^{2}$ is high for all used models in asymmetric dependence 0.2 (weak-dependence) and sample size $\mathrm{N}=15$, while the sample size is increasing, the $\mathrm{R}^{2}$ value exhibits a fluctuating case for all models used.

The highest $\mathrm{R}^{2}$ is seen in the logistic model in asymmetric dependence 0.2 (weakdependence) and sample size $\mathrm{N}=15, \mathrm{~N}=25$, and $\mathrm{N}=50$, while only in sample sizes $\mathrm{N}=100$ and $\mathrm{N}=150$, the highest $\mathrm{R}^{2}$ is seen in the Gompertz model with very little difference. On the other hand, in sample $\mathrm{N}=300$, the highest $\mathrm{R}^{2}$ has been seen in the Brody model. Because the parameters A, B, C are highly variable, no calculation has been made when the asymmetric dependence is 0.2

Table 3 (Continued). For Growth Models, Simulation Results when dependence is 0.2

$\begin{array}{llll}\text { Logistic } & \text { Gompertz } & \text { Brody } & \text { Von Bertalanffy }\end{array}$


Murat Gül, Mehmet Korkmaz

\begin{tabular}{lllll}
\hline Asymmetric & $R^{2}=0.10696$ & $R^{2}=0.10700$ & $R^{2}=0.10686$ & $R^{2}=0.10158$ \\
Dependence \\
Kendall Tau=0.2 \\
Clayton Copula \\
$\mathrm{N}=150$
\end{tabular}

From Table 3, for symmetric dependence, $\mathrm{R}^{2}$ values are high for all used models in symmetric dependence 0.2 (weak-dependence) and sample size $\mathrm{N}=15$. While the sample size is increasing, $\mathrm{R}^{2}$ values decrease for all models used.

The highest $\mathrm{R}^{2}$ is seen in the Logistic model in symmetric dependence 0.2(weakdependence) and sample size $\mathrm{N}=15, \mathrm{~N}=25, \mathrm{~N}=50, \mathrm{~N}=100$ and $\mathrm{N}=150$, while only in the sample size $\mathrm{N}=300$, the highest $\mathrm{R}^{2}$ is seen in the Von Bertalanffy with a little difference. Because the parameters $\mathrm{A}, \mathrm{B}, \mathrm{C}$ are highly variable, no calculation has been made when the symmetric dependence is 0.2

\section{Conclusion}

The aim of this study is to demonstrate the effect of dependence on some growth models. We used copulas to create dependence between variables. We examined the dependence between the dependent and the independent variable in terms of both symmetrical and asymmetric dependence. We calculated $\mathrm{R}^{2}$ values and parameters of Brody Von Bertalanffy, Logistic, Gompertz growth models. We compared the performance of the growth models used within the framework of this asymmetric and symmetric dependence. We discussed the main findings obtained in the simulation study.

Another important result is that the logistic model performs better in low sample size and weak dependence. When we look at the findings in more detail and take into account asymmetric and symmetric dependence, the Von Bertalanffy model has performed better, when the asymmetric dependence is 0.8 (strong- 
The Effect of Asymmetric and Symmetric Dependence in some Growth Models Using Copulas

dependence). The Brody model comes into prominence in symmetric dependence 0.8 (strong-dependence), while the Logistic model comes into prominence in symmetric dependence 0.2 (weak-dependence). Symmetric dependence 0.5 (medium-dependence) does not come forward with any model used.

Looking at the indirect results of our study, at levels of asymmetric and symmetric dependence of $0.8,0.5$ and 0.2 , that is, according to the degree of asymmetric and symmetric dependence, $\mathrm{R}^{2}$ values can be high, medium, low. That is to say, when asymmetric dependence is 0.8 , the value of $\mathrm{R}^{2}$ in all models used is quite high compared to other levels(weak, medium) of dependence, while the asymmetric dependence is 0.2 , the value of $\mathrm{R}^{2}$ in all models used is low compared to other levels(strong, medium) of dependence. In other words, asymmetric and symmetric dependence directly affects the value of $\mathrm{R}^{2}$ in all models used.

The contribution of this study to the literature is in two directions. The first is that even though correlation variables are used in many models, the effect on growth models has not been studied, including symmetric and asymmetric dependence. Secondly, it is about which growth model is better in strong, medium and weak correlated variables.

The results of our work will give an important idea of which model to apply when working with strong, medium, weak correlated data when they want to use growth models for practitioners. We've also found that dependence has changed the $\mathrm{R}^{2}$ and its parameters of growth models, as it has been shown in our study. Therefore, it will constitute a new perspective that they should be more careful when selecting variables, considering the effect of dependence before constructing the growth model.

\section{REFERENCES}

[1] Arnold, H.(2006), Dependence Modelling via the Copula Method; Vacation Student Project,CSIRO Mathematical and Information Sciences Macquarie University, 1-33;

[2] Chiang W.,Liu X.,Zhang T., Yang B. (2018), A Study of Exact Ridge Regression for Big Data; IEEE International Conference on Big Data, 978-15386-5035-6/18;

[3] Embrechts, P., Lindskog, F. and McNeil, A. (2003), Modeling Dependence With Copulas And Applications To Risk Management.In S.T. Rachev, editor, Handbook of Heavy Tailed Distribution in Finance, North Holland, Elsevier, 357360 ;

[4] English D., Milne M., Jacoby R., et al.(2005),The Effect of a School-Based Sun Protection Intervention on the Development of Melanocytic Nevi in Children: 6-Year Follow-up;Cancer Epidemiol Biomarkers Prevention ;14:977980;

DOI: 10.24818/18423264/55.1.21.15 
Murat Gül, Mehmet Korkmaz

[5]Gwaze D., Bridgwater F.E., Williams C.G. (2002),Genetic Analysis of Growth Curves for a Woody Perennial Species; Theoretical and Applied Genetics, 105: 526-531;

[6]Jiang L., Ke W. and Guofu Z. (2014),Asymmetry in Stock Returns: An Entropy Measure. http://ssrn.com, Working papers, Tsinghua University, Emory University, and Washington University in St. Louis;

[7]Kang J. (2020), Robust Estimation for Longitudinal Data Based upon Minimum Hellinger Distance; Journal of Applied Statistics, 47:1, 150-159; [8]Malevergne, Y., Sornette, D. (2003),Testing the Gaussian Copula Hypothesis for Financial Assets Dependencies.Quantitative Finance, 14:231-250;

[9]Manghi R.F., Cysneiros F.J, Paula G.A. (2019),Generalized Additive Partial Linear Models for Analyzing Correlated Data; Computational Statistics and Data Analysis, 129: 47-60;

[10]Nelsen, R.B. (2006), An Introduction to Copulas; Second Edition, Springer Series in Statistics , New York;

[11]Tsuyuguchi, A.B., Paula, G.A. \& Barros, M. (2019), Analysis of Correlated Birnbaum-Saunders Data Based on Estimating Equations; TEST, https://doi.org/10.1007/s11749-019-00675-1;

[12] Xanthakis V., Sullivanb L.M, Vasan R.S. (2013),Multilevel Modeling versus Cross-Sectional Analysis for Assessing the Longitudinal Tracking of Cardiovascular Risk Factors over Time; Statistics in Medicine, 32: 5028-5038; [13]Zwaigenbaum l., Young G., Stone W. et al. (2014),Early Head Growth in Infants at Risk of Autism: A Baby Siblings Research Consortium Study; Journal of the American Academy of Child \& Adolescent Psychiatry, Volume 53, Number 10, 1053-1062. 